\title{
Front Matter: Volume 7722
}

, "Front Matter: Volume 7722," Proc. SPIE 7722, Organic Photonics IV, 772201 (4 June 2010); doi: 10.1117/12.868864

SPIE. Event: SPIE Photonics Europe, 2010, Brussels, Belgium 


\title{
PROCEEDINGS OF SPIE
}

\section{Organic Photonics IV}

\author{
Paul L. Heremans \\ Reinder Coehoorn \\ Chihaya Adachi \\ Editors
}

12-15 April 2010

Brussels, Belgium

Sponsored by

SPIE

Cosponsored By

B-PHOT—Brussels Photonics Team (Belgium) - Brussels-Capital Region (Belgium) • FWO_Fonds Wetenschappelijk Onderzoek (Belgium) • ICO_International Commission for Optics $\bullet$ Ville de Bruxelles (Belgium)

Cooperating Organisations

CBO-BCO (Belgium) • EOS-European Optical Society (Germany) • IET-The Institution of Engineering and Technology (United Kingdom) • IOP_Institute of Physics (United Kingdom) Photonics4Life (Germany) • Photonics@be (Belgium) • Photonics 21 (Germany) • PromOptica (Belgium)

Published by

SPIE

Volume 7722 
The papers included in this volume were part of the technical conference cited on the cover and title page. Papers were selected and subject to review by the editors and conference program committee. Some conference presentations may not be available for publication. The papers published in these proceedings reflect the work and thoughts of the authors and are published herein as submitted. The publisher is not responsible for the validity of the information or for any outcomes resulting from reliance thereon.

Please use the following format to cite material from this book:

Author(s), "Title of Paper," in Organic Photonics IV, edited by Paul L. Heremans, Reinder Coehoorn, Chihaya Adachi, Proceedings of SPIE Vol. 7722 (SPIE, Bellingham, WA, 2010) Article CID Number.

ISSN 0277-786X

ISBN 9780819481955

Published by

SPIE

P.O. Box 10, Bellingham, Washington 98227-0010 USA

Telephone +1 3606763290 (Pacific Time) · Fax +1 3606471445

SPIE.org

Copyright (c) 2010, Society of Photo-Optical Instrumentation Engineers

Copying of material in this book for internal or personal use, or for the internal or personal use of specific clients, beyond the fair use provisions granted by the U.S. Copyright Law is authorized by SPIE subject to payment of copying fees. The Transactional Reporting Service base fee for this volume is $\$ 18.00$ per article (or portion thereof), which should be paid directly to the Copyright Clearance Center (CCC), 222 Rosewood Drive, Danvers, MA 01923. Payment may also be made electronically through CCC Online at copyright.com. Other copying for republication, resale, advertising or promotion, or any form of systematic or multiple reproduction of any material in this book is prohibited except with permission in writing from the publisher. The CCC fee code is $0277-786 \mathrm{X} / 10 / \$ 18.00$.

Printed in the United States of America.

Publication of record for individual papers is online in the SPIE Digital Library.

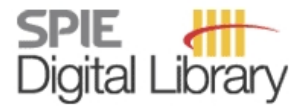

SPIEDigitalLibrary.org

Paper Numbering: Proceedings of SPIE follow an e-First publication model, with papers published first online and then in print and on CD-ROM. Papers are published as they are submitted and meet publication criteria. A unique, consistent, permanent citation identifier (CID) number is assigned to each article at the time of the first publication. Utilization of CIDs allows articles to be fully citable as soon they are published online, and connects the same identifier to all online, print, and electronic versions of the publication. SPIE uses a six-digit CID article numbering system in which:

- The first four digits correspond to the SPIE volume number.

- The last two digits indicate publication order within the volume using a Base 36 numbering system employing both numerals and letters. These two-number sets start with 00, 01, 02, 03, 04 , $05,06,07,08,09,0 A, 0 B \ldots 0 Z$, followed by 10-1Z, 20-2Z, etc.

The CID number appears on each page of the manuscript. The complete citation is used on the first page, and an abbreviated version on subsequent pages. Numbers in the index correspond to the last two digits of the six-digit CID number. 


\section{Contents}

ix Conference Committee

\section{OLED DEVICES AND APPLICATIONS I}

772202 White OLED devices and processes for lighting applications (Invited Paper) [7722-01] N. Ide, H. Tsuji, N. Ito, Y. Matsuhisa, S. Houzumi, T. Nishimori, Panasonic Electric Works Co., Ltd. (Japan)

\section{ORGANIC LASERS}

772207 Complementary pumping mechanisms of lambda-half organic microcavities [7722-06]

R. Brückner, M. Sudzius, V. G. Lyssenko, H. Fröb, K. Leo, Technische Univ. Dresden (Germany)

772208 Oblique angle lasing in a periodically pumped organic microcavity [7722-07]

S. I. Hintschich, V. G. Lyssenko, M. Sudzius, B. Schütte, H. Fröb, K. Leo, Technische Univ.

Dresden (Germany)

\section{OLED PHYSICS AND MODELLING I}

7722 OC Fundamental processes governing operation and degradation in state of the art P-OLEDs (Invited Paper) [7722-11]

M. Roberts, Cambridge Display Technology Ltd. (United Kingdom); K. Asada, Sumitomo Chemical Co., Ltd. (Japan); M. Cass, C. Coward, S. King, A. Lee, M. Pintani, M. Ramon, C. Foden, Cambridge Display Technology Ltd. (United Kingdom)

7722 OD Measuring the dipole orientation in OLEDs [7722-12] M. Flämmich, S. Roth, N. Danz, D. Michaelis, A. H. Bräuer, Fraunhofer-Institut für Angewandte Optik und Feinmechanik (Germany); M. C. Gather, K. Meerholz, Univ. zu Köln (Germany)

7722 OF The influence of the optical environment on the shape of the emission profile and methods of its determination [7722-14]

B. Perucco, FLUXiM AG (Switzerland); N. A. Reinke, Zurich Univ. of Applied Sciences

(Switzerland); F. Müller, D. Rezzonico, FLUXiM AG (Switzerland); B. Ruhstaller, FLUXiM AG

(Switzerland) and Zurich Univ. of Applied Sciences (Switzerland)

\section{OPV DEVICES}

7722 OG Efficient and long-term stable organic vacuum deposited tandem solar cells (Invited Paper) [7722-15]

C. L. Uhrich, G. Schwartz, B. Maennig, W. M. Gnehr, S. Sonntag, O. Erfurth, E. Wollrab, K. Walzer, J. Foerster, A. Weiss, O. Tsaryova, heliatek GmbH (Germany); K. Leo, M. K. Riede, Technische Univ. Dresden (Germany); M. Pfeiffer, heliatek GmbH (Germany) 
7722 OK Comparison of different conditions for accelerated ageing of small molecule organic solar cells [7722-19]

M. Hermenau, K. Leo, M. Riede, Technische Univ. Dresden (Germany)

\section{OLED DEVICES AND APPLICATIONS II}

7722 OP Towards efficient next generation light sources: combined solution processed and evaporated layers for OLEDs (Invited Paper) [7722-24]

D. Hartmann, W. Sarfert, Siemens AG (Germany); S. Meier, Siemens AG (Germany) and Univ. Erlangen-Nuremberg (Germany); H. Bolink, S. García Santamaría, Univ. de Valencia (Spain); J. Wecker, Siemens AG (Germany)

7722 OR Highly efficient inverted top-emitting organic electroluminescent devices with doped charge transport layers [7722-26]

M. Thomschke, M. Furno, B. Lüssem, K. Leo, Technische Univ. Dresden (Germany)

\section{OPV PHYSICS AND MODELLING II}

7722 OT Charge carrier mobility in disordered organic blends [7722-28]

L. J. A. Koster, Eindhoven Univ. of Technology (Netherlands)

$77220 \mathrm{~V}$ Transient photocurrent response of organic bulk heterojunction solar cells [7722-30] M. T. Neukom, N. A. Reinke, K. A. Brossi, Zurich Univ. of Applied Sciences (Switzerland); B. Ruhstaller, Zurich Univ. of Applied Sciences (Switzerland) and FLUXiM AG (Switzerland)

MATERIALS AND PHOTONIC-ENHANCED MATERIALS I

7722 OY Photoluminescence enhancement of semiconducting-carbon-nanotubes-based thin films [7722-33]

E. Gaufres, N. Izard, X. Le Roux, D. Marris-Morini, Institut d'Électronique Fondamentale, CNRS, Univ. Paris-Sud (France); S. Kazaoui, National Institute of Advanced Industrial Science and Technology (Japan); E. Cassan, L. Vivien, Institut d'Électronique Fondamentale, CNRS, Univ. Paris-Sud (France)

\section{OPV PHYSICS AND MODELLING II}

772214 Modelling exciton diffusion in disordered conjugated polymers [7722-39]

S. Athanasopoulos, E. V. Emelianova, Univ. de Mons-Hainaut (Belgium); A. B. Walker, Univ. of Bath (United Kingdom); D. Beljonne, Univ. de Mons-Hainaut (Belgium)

POSTER SESSION: ORGANIC LIGHT EMITTING DIODES

772218 Device history dependent effects in dark injection transient current measurements of charge mobility in organic light-emitting diodes [7722-43]

S. Knox, National Physical Lab. (United Kingdom); H. Jones, Univ. of Southampton (United Kingdom); T. Esward, National Physical Lab. (United Kingdom) 
7722 IB IR studies on the interaction of $\mathrm{Ca}$ and $\mathbf{M g}$ with the blue emitter material $\operatorname{Ir}(\mathrm{cn}-\mathrm{pmbic})_{3}$ [7722-46]

T. Glaser, M. Binder, A. Pucci, Ruprecht-Karls-Univ. Heidelberg (Germany); C. Schildknecht,

C. Lennartz, BASF SE (Germany)

7722 1D White organic light-emitting diodes with top-emitting structure for high color quality and forward-directed light emission [7722-48]

P. Freitag, S. Reineke, M. Furno, B. Lüssem, K. Leo, Technische Univ. Dresden (Germany)

POSTER SESSION: ORGANIC LASER STRUCTURES AND APPLICATIONS

7722 1G Second-order distributed feedback lasers based on films containing perylenediimide derivatives [7722-51]

V. Navarro-Fuster, P. G. Boj, J. M. Villalvilla, J. A. Quintana, M. A. Díaz-García, Univ. de Alicante (Spain); V. Trabadelo, EMPA-Swiss Federal Labs. for Materials Testing and Research (Switzerland) and Tekniker (Spain); A. Juarros, A. Retolaza, S. Merino, Tekniker (Spain)

$77221 \mathrm{H} \quad$ Azobenzene-based surface relief gratings for thin film distributed feedback lasers [7722-52] S. Döring, T. Rabe, R. Rosenhaver, O. Kulikovska, N. Hildebrandt, J. Stumpe, FraunhoferInstitut für Angewandte Polymerforschung (Germany)

$772211 \quad$ Optical spectroscopy with organic semiconductor lasers (Best Student Paper Award) [7722-53]

S. Klinkhammer, T. Woggon, C. Vannahme, Karlsruhe Institute of Technology (Germany);

U. Geyer, Karlsruhe Institute of Technology (Germany) and Christian-Albrechts-Univ. Kiel

(Germany); T. Mappes, U. Lemmer, Karlsruhe Institute of Technology (Germany)

POSTER SESSION: ORGANIC PHOTOVOLTAICS

$77221 \mathrm{~L} \quad$ Nano-structure control in the bulk heterojunction layer for organic solar cells [7722-57] D. Yamanaka, S. Watanabe, T. Mizutani, K. Kojima, S. Ochiai, Aichi Institute of Technology (Japan)

$77221 \mathrm{M} \quad$ Fabrication and performance of organic thin film solar cells using a painting method [7722-58]

S. Ochiai, H. Ishihara, T. Mizutani, K. Kojima, Aichi Institute of Technology (Japan)

$77221 \mathrm{~N} \quad$ Organic solar cell performance and nano-morphology of poly(3-hexylthiophene-2,5-diyl) (P3HT)/PCBM thin film using poly(3-ocxylthiophene) [7722-59]

K. Sakai, K. Kojima, T. Mizutani, S. Ochiai, Aichi Institute of Technology (Japan)

772210 Island size effects in organic optoelectronic devices [7722-60]

M. Nguyen, A. Z. Turak, F. Maye, J. Heidkamp, Max-Planck-Institut für Metallforschung (Germany); J. Wrachtrup, Univ. Stuttgart (Germany); H. Dosch, Deutsches ElektronenSynchrotron (Germany)

$77221 \mathrm{P}$ The appropriateness of organic solar cells for indoor lighting conditions [7722-61] B. Minnaert, P. Veelaert, Univ. College Ghent (Belgium) 
$77221 \mathrm{U}$ Photo-induced processes in new materials for electro-optical applications [7722-67] R. Siebert, Friedrich-Schiller-Univ. Jena (Germany); A. Winter, Eindhoven Univ. of Technology (Netherlands); U. S. Schubert, Eindhoven Univ. of Technology (Netherlands) and Friedrich-Schiller-Univ. Jena (Germany); M. Schmitt, Friedrich-Schiller-Univ. Jena (Germany); B. Dietzek, J. Popp, Friedrich-Schiller-Univ. Jena (Germany) and Institut für Photonische Technologien e.V. (Germany)

772220 Influence of particle plasmon resonance on the photoluminescence of organic semiconductor blend [7722-73]

F. Dou, C. Peng, H. Liu, J. Wang, S. Feng, X. Zhang, Beijing Univ. of Technology (China)

POSTER SESSION: NONLINEAR OPTICS, HOLOGRAPHY, AND OPTICAL STORAGE

$772221 \quad$ Polymer composites containing photochromic dye solution [7722-74]

M. Saito, K. Sakiyama, R. Mochizuki, K. Ohashi, Ryukoku Univ. (Japan)

772222 Initiator system in holographic photopolymer materials [7722-75]

M. Ortuño, E. Fernández, R. Fuentes, S. Gallego, A. Márquez, Univ. de Alicante (Spain)

772224 Towards a unifying theory for the first-, second-, and third-order molecular (non)linear optical response [7722-77]

J. Pérez-Moreno, Katholieke Univ. Leuven (Belgium); K. Clays, Katholieke Univ. Leuven (Belgium) and Washington State Univ. (United States); M. G. Kuzyk, Washington State Univ. (United States)

772225 Light-sensitive organic systems and multilayer polymer structures for optical recording media [7722-78]

V. A. Barachevsky, O. I. Kobeleva, T. M. Valova, A. O. Ait, A. A. Dunaev, A. M. Gorelik, Photochemistry Ctr. (Russian Federation); M. M. Krayushkin, K. S. Levchenko,

V. N. Yarovenko, Zelinsky Institute of Organic Chemistry (Russian Federation); V. V. Kyiko, Prokhorov General Physics Institute (Russian Federation); E. H. Grebennikov, Technomash, Ltd. (Russian Federation)

772226 Multiphoton absorption in polydiacetylenes adsorbed on metal nanostructures [7722-79] R. Pilot, R. Bozio, Consorzio INSTM (Italy) and Univ. degli Studi di Padova (Italy); A. Demartini, M. Alloisio, G. Dellepiane, Consorzio INSTM (Italy) and Univ. degli Studi di Genova (Italy);

E. Giorgetti, Consorzio INSTM (Italy) and ISC-CNR (Italy)

772228 Second-order nonlinear optical properties of zwitterionic chromophores [7722-81] A. Teshome, I. Asselberghs, Katholieke Univ. Leuven (Belgium); D. J. Clarke, Industrial Research Ltd. (New Zealand); A. P. Middleton, Victoria Univ. of Wellington (New Zealand); M. D. H. Bhuiyan, Industrial Research Ltd. (New Zealand); G. J. Smith, Victoria Univ. of Wellington (New Zealand); A. J. Kay, Industrial Research Ltd. (New Zealand); K. Clays, Katholieke Univ. Leuven (Belgium)

772229 Low polymerization-shrinkage nanoparticle-polymer composite films based on thiol-ene photopolymerization for holographic data storage [7722-82]

Y. Tomita, E. Hata, K. Omura, S. Yasui, The Univ. of Electro-Communications (Japan) 
7722 2A Z-scan characterization of nonlinear optical effects in polymer films incorporating hyperbranched polymer-metallic nanoparticle complex [7722-83]

X. Liu, Y. Tomita, The Univ. of Electro-Communications (Japan); K. Yasui, K. Kojima, K. Chikama, Nissan Chemical Industries, Ltd. (Japan)

Author Index 
Downloaded From: https://www.spiedigitallibrary.org/conference-proceedings-of-spie on 26 Apr 2023

Terms of Use: https://www.spiedigitallibrary.org/terms-of-use 


\title{
Conference Committee
}

\author{
Symposium Chairs \\ Francis Berghmans, Vrije Universiteit Brussel (Belgium) \\ Ronan Burgess, European Commission (Belgium) \\ Jürgen Popp, Institut für Photonische Technologien e.V. (Germany) \\ Peter Hartmann, SCHOTT AG (Germany) \\ Hugo Thienpont, Vrije Universiteit Brussel (Belgium)
}

Conference Chairs

Paul L. Heremans, IMEC (Belgium)

Reinder Coehoorn, Philips Research Nederland B.V. (Netherlands)

Chihaya Adachi, Kyushu University (Japan)

Program Committee

Heinrich Becker, Merck OLED Materials GmbH (Germany)

David Beljonne, Université de Mons-Hainaut (Belgium)

Paul W. M. Blom, TNO Science and Industry (Netherlands)

Herbert F. Boerner, Philips Research (Germany)

Donal D. C. Bradley, Imperial College London (United Kingdom)

Franco Cacialli, University College London (United Kingdom)

Gunther Haas, MicroOLED (France)

Alan J. Heeger, University of California, Santa Barbara (United States)

Richard H. Friend, University of Cambridge (United Kingdom)

Rene A. Janssen, Technische Universiteit Eindhoven (Netherlands)

Junji Kido, Yamagata University (Japan)

Guglielmo Lanzani, Politecnico di Milano (Italy)

Uli Lemmer, Universität Karlsruhe (Germany)

Karl Leo, Technische Universität Dresden (Germany)

Rainer F. Mahrt, IBM Zürich Research Laboratory (Switzerland)

William R. Salaneck, Linköping Universitet (Sweden)

Niyazi Serdar Sariciffci, Johannes Kepler Universität Linz (Austria)

Paul van der Schaaf, Ciba Specialty Chemicals Holding, Inc.

(Switzerland)

\section{Session Chairs}

1 OLED Devices and Applications I

Paul L. Heremans, IMEC (Belgium)

2 Organic Lasers

Chihaya Adachi, Kyushu University (Japan) 
OLED Physics and Modelling I

Reinder Coehoorn, Philips Research Nederland B.V. (Netherlands)

4 OPV Devices

David Hartmann, Siemens AG (Germany)

5 OLED Physics and Modelling II

Matthew Roberts, Cambridge Display Technology Ltd.

(United Kingdom)

6 OLED Devices and Applications II

Lambert Jan Anton Koster, Technische Universiteit Eindhoven (Netherlands)

$7 \quad$ OPV Physics and Modelling II

Wolfgang Brütting, Universität Augsburg (Germany)

8 Materials and Photonic-Enhanced Materials I

Michele Muccini, Istituto per lo Studio dei Materiali Nanostrutturati (Italy)

9 Materials and Photonic-Enhanced Materials II

Antonio F. Facchetti, Polyera Corporation (United States)

10 OPV Physics and Modelling II

Paul L. Heremans, IMEC (Belgium) 